\title{
A Novel Epitope on the C-Terminus of SmD1 Is Recognized by the Majority of Sera from Patients with Systemic Lupus Erythematosus
}

\author{
Gabriela Riemekasten, ${ }^{*}$ Jeannette Marell,, ${ }^{\star}$ Grit Trebeljahr, ${ }^{*}$ Rolf Klein,, ${ }^{*}$ Gert Hausdorf, ${ }^{*}$ Thomas Häupl," \\ Jens Schneider-Mergener, ${ }^{\ddagger}$ Gerd R. Burmester, ${ }^{\star}$ and Falk Hiepe* \\ *Department of Medicine, Rheumatology and Clinical Immunology and ${ }^{\ddagger}$ Institute of Medical Immunology, Charité University Hospital, \\ Humboldt University at Berlin, D-10117 Berlin, Germany
}

\begin{abstract}
The SmD1 protein is a specific target for the autoantibody response in SLE. To further analyze this reactivity epitope, mapping was performed with cellulose-bound 13-mer peptides overlapping 10 amino acids (aa). In this initial approach, 4 out of 15 SLE sera recognized more than five overlapping peptides of the SmD1 C-terminus. Therefore, longer oligopeptides of up to 37 aa of this region were generated and probed for as antigens by ELISA. For the SmD1 aa 83-119 polypeptide, there was a striking increase of reactivity with $70.0 \%$ positive reactions out of 167 SLE sera. In contrast, 105 healthy control sera were negative, and only $8.3 \%$ of sera from patients with other inflammatory diseases ( $n=267)$ exhibited a response, which was of low level only. The anti-SmD1 $1_{83-119}$ reactivity was significantly higher in anti-dsDNA antibody positive vs. negative sera $(P<0.001)$ and correlated with disease activity. Four of five human monoclonal anti-dsDNA antibodies also reacted with $\mathrm{SmD1}_{83-119}$. The specificity for SmD1 was demonstrated by inhibition experiments and immunization of rabbits with $\mathrm{SmD1}_{83-119}$ inducing SmD1-specific antibodies. In conclusion, the SmD1 $1_{83-119}$ peptide was demonstrated to be an important and highly specific target of the autoimmune response in SLE. The high sensitivity of this ELISA probably depends on a conformational epitope, which appears not to be accessible in the full-size SmD1 protein. (J. Clin. Invest. 1998. 102:754-763.) Key words: SmD1 peptides • epitope mapping • autoantibodies • systemic lupus erythematosus • immunization
\end{abstract}

\section{Introduction}

Systemic lupus erythematosus (SLE) is an autoimmune disease that is characterized by the production of autoantibodies directed against a wide spectrum of nuclear, cytoplasmic, and

Address correspondence to Gabriela Riemekasten, M.D., Department of Medicine, Rheumatology and Clinical Immunology, Charité University Hospital, Schumannstr. 20/21, D-10117 Berlin, Germany. Phone: 49-30-28028408; FAX: 49-30-28028082; E-mail: hiepe@rz. charite.hu-berlin.de

Received for publication 6 January 1998 and accepted in revised form 4 June 1998.

J. Clin. Invest.

(C) The American Society for Clinical Investigation, Inc. 0021-9738/98/08/0754/10 \$2.00

Volume 102, Number 4, August 1998, 754-763

http://www.jci.org cell membrane autoantigens, including DNA, Sm, PCNA, Ku, U1RNP, nucleosomes, histones, Ro/SS-A, La/SS-B, ribosomal RNP, phospholipids, Hsp 90, and proteasomes (1). Beside the anti-dsDNA antibodies, the anti-Sm antibodies, first described by Tan and Kunkel (2), are specific for SLE and thus have been included as one of the American College of Rheumatology classification criteria for this disease (3). They are not only a diagnostic marker but also a reliable measure of disease activity in SLE (4). Furthermore, anti-Sm antibodies are associated with renal involvement, serositis, and lung fibrosis $(4,5)$. However, their frequency in SLE patients is rather low and varies between 5 and $30 \%$ depending on the serologic tests used and the ethnic origin of the patient population $(6,7)$.

The Sm antigen was discovered to be part of the spliceosomal complex that plays essential roles in RNA processing (810). It comprises at least nine different polypeptides termed according to their electrophoretic mobility on SDS-PAGE B (B1, $28 \mathrm{kDa}), \mathrm{B}^{\prime}$ (B2, $\left.29 \mathrm{kDa}\right), \mathrm{N}$ (B3, $\left.29.5 \mathrm{kDa}\right), \mathrm{D} 1$ (16 kDa), D2 (16.5 kDa), D3 (18 kDa), E (12 kDa), F (11 kDa), and G (9 $\mathrm{kDa}$ ) representing the core proteins of the $\mathrm{U} 1, \mathrm{U} 2, \mathrm{U} 4, \mathrm{U} 5$, U6, U7, U11, and U12 small nuclear ribonucleoproteins $(\text { RNPs })^{1}(11,12)$. All of these core proteins can serve as targets of the anti-Sm antibodies, especially the B polypeptides and the D1 polypeptide, which are considered to be the major target of anti-Sm reactivity $(13,14)$. Since anti-U1RNP sera can also react, to a variable extent, with B and $\mathrm{B}^{\prime}$ proteins (15), the D1 protein appears to be the most important $\mathrm{Sm}$ antigen. Therefore, $\mathrm{SmD} 1$ is a crucial subject to investigate regarding its molecular structure and immunodominant epitopes. On the basis of the published sequence of SmD1 (16), we performed a linear epitope mapping using cellulose-bound peptides resulting in a C-terminal immunoreactive domain of SmD1. As a result, peptides of different lengths of this immunodominant region were synthesized and used in ELISA. Surprisingly, the longest $\mathrm{SmD} 1$ peptide synthesized that consisted of the amino acid sequence from amino acid (aa) 83 to aa 119 was specifically recognized by the majority of sera from patients with SLE in ELISA. The frequency of reactivity directed against this $\mathrm{SmD} 1$ peptide was markedly higher than in other tests, including immunoblotting with cell extract and ELISA, using affinity purified $\mathrm{SmD}$ antigen. These results show that a critical autoantigenic epitope of SmD1 is C-terminal, probably depends on conformation, and only forms in peptides of critical length. The high sensitivity of the ELISA targeting the SmD1 $1_{83-119}$ peptide in contrast to the usual anti-SmD assays suggests the detection of a hidden epitope.

1. Abbreviations used in this paper: aa, amino acid; AU, arbitrary units; CIE, counterimmunoelectrophoresis; MCTD, mixed connective tissue disease; PBC, primary biliary cirrhosis; PBS-T, PBS 0.1\% Tween 20; RNP, ribonucleoproteins; SB, sample buffer; SS, Sjögren's syndrome; SSc, systemic sclerosis. 


\section{Methods}

Sera. Sera were collected from 167 patients with SLE, 15 with primary Sjögren's syndrome (SS), 23 with mixed connective tissue disease (MCTD), 20 with systemic sclerosis (SSc), 28 with primary biliary cirrhosis (PBC), 28 with RA, 88 with HIV infection, 15 with ulcerative colitis, 20 with hepatitis B infection, 30 with Lyme disease, and 105 healthy subjects and stored at $-20^{\circ} \mathrm{C}$. Diagnosis of SLE (3), RA (17), and SSc (18) was established according to the respective criteria of the American College of Rheumatology, whereas the other diseases were identified upon typical clinical and laboratory findings. 15 SLE sera were analyzed in all available tests including dot assay for linear SmD1 epitopes, immunoblotting, counterimmunoelectrophoresis (CIE), a commercial anti-Sm-ELISA (Sm-Elias; Elias, Freiburg, Germany), and the SmD1 $1_{83-119}$ ELISA, as described later. For longitudinal studies, sera of six SLE patients with acute onset of SLE or exacerbation of a known SLE were investigated during a period of 6 to $12 \mathrm{mo}$

Monoclonal antibodies and polyclonal sera. The mouse antiSmD1 monoclonal IgA antibody derived from culture supernants of clone ANA 127 (Quartett, Berlin, Germany) was included in these experiments. Polyclonal sera from rabbits immunized with SmD1, D2, and D3 were kindly provided by $\mathrm{H}$. Brahms and R. Lührmann (Marburg, Germany).

Five well-characterized human hybridomas secreting anti-dsDNA IgG antibodies derived from three SLE patients (32.B9, 33.F12, 33.C9, 33H11, and 35.21) were kindly provided by Winkler et al. (19). Antibody preparations were diluted 1:10 in sample buffer (SB, 1\% dry non-fat milk in PBS, $0.1 \%$ Tween 20 ) and tested for their reactivity against the $S \mathrm{SmD} 1_{83-119}$ peptide by ELISA, as described later.

Epitope mapping. As shown in Table I, 37 overlapping peptides were simultaneously synthesized covalently bound to a continuous cellulose membrane (Whatmann 540 paper; Whatman, Maidstone, UK), as described in detail below (20) (Table I).

Similarly to a dot blot assay, 15 SLE and 2 human control sera were diluted 1:100 and incubated with the cellulose-bound peptides for $2 \mathrm{~h}$ at room temperature. After washing with PBS $0.1 \%$ Tween 20 (PBS-T), the reaction was visualized with 1:1,000 diluted alkaline phosphatase-conjugated anti-human IgG (Sigma Chemical Co., St. Louis, MO) followed by incubation with BCIP-NBT (5-bromo4-chloro-3-indolyl phosphate nitroblue tetrazolium; Sigma).

Analysis of the reactivity against $C$-terminal synthetic peptides of different length. To characterize the reactivity against the two SmD1 sequence regions recognized by SLE sera, five peptides of different length were synthesized as free peptides according to the protocol described by Atherton (21) and used as antigens in the ELISA: (1.) 83-99 aa, VEPKVKSKKREAVAGRG; (2.) 105-119 aa, GRGRGRGR-
GRGGPRR; (3.) 95-119 aa, VAGRGRGRGRGRGRGRGRGRGGPRR; (4.) 83-119 aa, VEPKVKSKKREAVAGRGRGRGRGRGRGRGRGRGGPRR; (5.) 34-46 aa, NTHLKAVKMTLKN.

Reactivity against these peptides was tested in 30 SLE sera and 10 sera from healthy donors. Purity of $S m D 1_{83-119}$ peptide was confirmed by HPLC, showing a single peak.

SmD1 peptide ELISA. Polystyrene well plates (Nunc, Roskilde, Denmark) were coated with $0.5 \mu \mathrm{g}$ of the individual peptides dissolved in $50 \mu$ l of coating buffer ( $0.05 \mathrm{M}$ carbonate buffer, $\mathrm{pH} 9.5)$. After incubation for $6 \mathrm{~h}$ at room temperature, three washes in PBS-T were performed, and nonspecific binding sites were blocked for $1 \mathrm{~h}$ at room temperature by using SB. Patient sera were diluted 1:100 in SB and added to the wells for $2 \mathrm{~h}$ at room temperature. After repeated washings, horseradish peroxidase conjugated rabbit anti-human IgG $(1 \mu \mathrm{g} / \mathrm{ml}$; IMTEC, Zepernick, Germany) were added for $2 \mathrm{~h}$ at room temperature. The final reaction was visualized by $5 \mathrm{mmol} / \mathrm{l} o$-phenylenediamine (OPD; Merck, Darmstadt, Germany) in the presence of $5 \mathrm{mmol} / 1 \mathrm{H}_{2} \mathrm{O}_{2}$ in $0.1 \mathrm{~mol} / \mathrm{l}$ citrate buffer ( $\mathrm{pH} 5.5$ ) by incubation for 5-10 min at room temperature. The reaction was terminated with $1 \mathrm{~mol} / 1 \mathrm{H}_{2} \mathrm{SO}_{4}$ and $0.05 \mathrm{~mol} / 1 \mathrm{Na}_{2} \mathrm{SO}_{3}$. OD values were measured at $492 \mathrm{~nm}$ by multiscan (Anthos Reader 2001; Anthos Labtech Instruments, Salzburg, Austria).

A standard curve was established for each assay by using a highly reactive serum from one specific SLE patient. Reactivity of this serum at a dilution of 1:100 was defined as 10,000 arbitrary units (AU). The upper limit of the normal range was defined as mean value $+2 \mathrm{SD}$ of the reactivities in 105 normal human sera (dilution 1:100). Thus, for the $\mathrm{SmD} 1_{83-119}$ peptide, the cutoff was 223 units. Each serum was assayed in duplicates. Sera with anti-SmD1 $1_{83-119}$ response above 10,000 AU were diluted up to 1:400 to determine exact anti-SmD1 $1_{83-119}$ reactivity levels.

CIE. Conventional CIE using rabbit thymus extract was performed as described (22). Standard sera were kindly provided by the Centers for Disease Control (Atlanta, GA).

Anti-dsDNA-ELISA. Anti-dsDNA antibodies were measured by ELISA as described previously (23). In brief, methylated BSAprecoated and dsDNA-coated microtiter plates were incubated with patient sera for the detection of IgG and IgM anti-dsDNA autoantibodies.

Western blot assay. Immunoblotting was performed as reference method, using MOLT four-cell extracts and $7.5 \mu \mathrm{g} /$ well of recombinant SmD1 protein as described later according to standard protocols with minor modifications (24). For electrophoretic separation of SmD1, 2, and 3 in individual bands, a high TEMED polyacrylamide gel was used, as described by Lehmeier et al. (11). After blocking with SB for $1 \mathrm{~h}$ at room temperature, nitrocellulose strips were incubated with patient sera diluted 1:100 in SB for $2 \mathrm{~h}$ followed by wash-

Table I.

\begin{tabular}{|c|c|c|c|c|c|}
\hline \multicolumn{6}{|c|}{ Overlapping peptides } \\
\hline MKLVRFLMKLSHE & (1) & LKAVKMTLKNREP & (14) & LDTIRVDVEPKVK & (26) \\
\hline VRFLMKLSHETVT & (2) & VKMTLKNREPVQL & $(15)$ & IRVDVEPKVKSKK & (27) \\
\hline LMKLSHETVTIEL & (3) & TLKNREPVQLETL & (16) & VDVEPKVKSKKRE & (28) \\
\hline LSHETVTIELKNG & (4) & NREPVQLETLSIR & (17) & VEPKVKSKKREAV & (29) \\
\hline ETVTIELKNGTQV & (5) & PVQLETLSIRGNR & (18) & KVKSKKREAVAGR & (30) \\
\hline TIELKNGTQVHGT & (6) & LETLSIRGNRIRY & (19) & SKKREAVAGRGRG & (31) \\
\hline LKNGTQVHGTITG & (7) & LSIRGNRIRYFIL & $(20)$ & REAVAGRGRGRGR & (32) \\
\hline GTQVHGTITGVDV & (8) & RGNRIRYFILPDS & (21) & VAGRGRGRGRGRG & (33) \\
\hline VHGTITGVDVSMN & (9) & RIRYFILPDSLPL & $(22)$ & RGRGRGRGRGRGR & (34) \\
\hline TITGVDVSMNTHL & (10) & YFILPDSLPLDTI & (23) & GRGRGRGRGRGRG & (35) \\
\hline GVDVSMNTHLKAV & (11) & LPDSLPLDTIRVD & (24) & RGRGRGRGRGRGG & (36) \\
\hline VSMNTHLKAVKMT & (12) & SLPLDTIRVDVEP & $(25)$ & GRGRGRGRGGPRR & (37) \\
\hline
\end{tabular}


ing in PBS-T for 45 min with three changes. Bound $\operatorname{IgG}$ was detected with horseradish peroxidase conjugated rabbit anti-human IgG followed by chemiluminescence by using ECL reagents (Amersham Life Sciences, Arlington Heights, IL) as described (25).

Recombinant SmD1 protein. For expression of human SmD1 full-length protein, the corresponding cDNA was amplified by PCR (26) using the synthetic oligonucleotides hSmD1-P1 (5'-CGCCGGATCCATGAAGCTGTGAG-3') and hSmD1-P2 (5'CTTGAAGATCTTTATCGCCTAGGAC-3'). BamHI and BgIII sites (underlined) were introduced, flanking the coding sequence of human SmD1. A cDNA preparation from human monocytes was used as template, and the amplification reaction was performed in a thermal cycler (PTC200; MJ Research, Watertown, MA, Biozym). The amplification product $(380 \mathrm{bp})$ was cloned into the pGEM-T vector (Promega, Madison, WI) and was analyzed by automated sequencing (ALF; Pharmacia, Uppsala, Sweden). The expression plasmid pQSmD1 was constructed with a fragment obtained by restriction digestion of the pGEM-SmD1 at the BamHI site and within the polylinker sequences of the vector at the PstI site. The fragment was cloned into the expression vector pQE-31W (Qiagen) linearized with BamHI and PstI.

Expression and purification of recombinant human SmD1. Overnight cultures of Escherichia coli M15 (pREP4) transformed with the expression plasmid pQSmD1 were grown at $37^{\circ} \mathrm{C}$ in LB medium supplemented with $150 \mu \mathrm{g} / \mathrm{ml}$ ampicillin and $30 \mu \mathrm{g} / \mathrm{ml}$ kanamycin (27). After 1:100 dilution and incubation to an optical density of 0.8 at $37^{\circ} \mathrm{C}$, the cultures were induced with $2 \mathrm{mM}$ isopropyl- $\beta$-thiogalactosidase and allowed to grow for another 3 h. E. coli was collected by centrifugation and lysis was perfomed in $5 \mathrm{ml}$ of $6 \mathrm{M}$ guanidine hydrochloride and $10 \mathrm{mM}$ TRIS- $\mathrm{HCl}$ at $\mathrm{pH}$ 8.0. Recombinant proteins were purified in a single step by using $6 \times$ His/Ni-NTA columns (28) and denaturing conditions as outlined in the manufacturer's description (Pharmacia). The SmD1 containing elution fraction was dialyzed in PBS ( $\mathrm{pH}$ 7.4) and used as antigen for immunoblotting.

Immunization of rabbits with $S m D 1_{83-119}$ peptide. The $\mathrm{SmD} 1_{83-119}$ peptide was coupled to keyhole limpet hemocyanin by disulfide linkage with the use of cystamine as described (29). Three rabbits were immunized with $500 \mu \mathrm{g} / \mathrm{ml}$ of keyhole limpet hemocyanin-coupled $S m D 1_{83-119}$ peptide in complete Freund's adjuvant and boostered with $250 \mu \mathrm{g} / \mathrm{ml}$ of this haptene-protein conjugate in incomplete Freund's adjuvant every $14 \mathrm{~d}$ up to 3 mo. Development of an antibody response was measured by the anti-SmD1 $1_{83-119}$ peptide ELISA and by immunoblotting using MOLT4 and recombinant SmD1 protein. Each serum was diluted up to 1:20,000 in sample buffer and tested before and after immunization. The reactivity was measured as OD.

Competition assays. For removal of anti-SmD1 specific antibodies in SmD positive SLE sera, adsorption was performed with polystyrene-bound $\mathrm{SmD}_{83-119}$ peptide, free $\mathrm{SmD} 1_{83-119}$ peptide, and TALON-bound recombinant SmD1. In detail, $50 \mu$ l of 1:100 diluted serum was sequentially incubated up to eight times in SmD1 $1_{83-119^{-}}$ coated ELISA wells for $30 \mathrm{~min}$ per step. As control for unspecific antibody adsorption, diluted SLE serum was applied similarly to uncoated polystyrene wells. Incubation with free peptide or oxypolygelatine (Gelafusal; Serumwerke Bernburg, Germany) as a control was performed in $500 \mu \mathrm{l}$ for $1 \mathrm{~h}$, using 1:100 diluted serum and $80 \mu \mathrm{g} / \mathrm{ml}$ of the respective protein. For adsorption to recombinant SmD1, the protein was bound to a 50- $\mu 1$ TALON metal affinity resin (Clontech, Palo Alto, CA), and patient sera diluted 1:50 were incubated with this resin in $500 \mu \mathrm{l}$ volume for $1 \mathrm{~h}$. All adsorbed sera and individual controls were analyzed by immunoblotting with MOLT4 cell extracts or recombinant SmD1 protein as well as $\mathrm{SmD} 1_{83-119}$ peptide ELISA.

Statistics. The Mann-Whitney U-test was applied for statistical analysis of an association between anti-dsDNA and anti-SmD1 $1_{83-119}$ antibodies.

\section{Results}

Epitope mapping with 13-mer peptides of SmD1 by dot assay. To determine important regions of the SmD1 protein detected by SLE autoantibodies, 15 SLE sera and 2 human control sera were tested for reactivity against 37 cellulose-bound consecutive linear peptides of 13-aa length and 10-aa overlap. 4 of 15 SLE sera $(27 \%)$ showed significant reactions predominantly against the region 83-99 aa. Two of these four sera also interacted with peptides spanning amino acids 34-46. One representative dot assay result is shown in Fig. 1. In this patient, high reactivity against two regions associated with weaker staining of neighboring peptides suggests an epitope formed by sequences including more than one single 13-aa peptide. Sera of two normal donors did not react with any of the cellulose-bound SmD1 peptides.

Characterization of the SmD1-C-terminal epitope with 17mer to 37-mer peptides. 13-aa as well as 17-aa soluble peptides of the two major sites recognized by linear epitope mapping (amino acid region 34-46 and 83-99) were synthesized as described above according to Atherton et al. (21). An ELISA system was established, using these peptides as antigens. 10 sera from normal donors were screened to determine the cutoff. Analysis of 30 SLE sera including the 15 sera tested in the linear epitope mapping revealed no significant difference to normal donor values (Fig. 2). Therefore, three longer peptides of the SmD1-C-terminus aa 105-119, 95-119, and 83-119 were synthesized and rescreened with the same sera. This revealed positive reactions in five patients $(17 \%)$ for peptide $S m D 1_{105-119}$, $11(37 \%)$ for $\mathrm{SmD}_{95-119}$, and 19 (63\%) for $\mathrm{SmD}_{83-119}$ (Fig. 2 ). Double coating of wells with $\mathrm{SmD} 1_{83-99} / \mathrm{SmD} 1_{95-119}$ or $\mathrm{SmD} 1_{83-99} / \mathrm{SmD}_{105-119}$ did not increase reactivity above $25 \%$, demonstrating that only the uninterrupted $S m D 1_{83-119}$ peptide is sufficiently recognized by SLE autoantibodies (data not shown). Further characterization of the region around aa 3446 with similarly increased peptide sizes (28-61 aa sequence) did not show increased reactivity in SLE sera.

Sensitivity and specificity of anti-SmD1 $1_{83-119}$ antibodies. Sera from 167 SLE patients, 267 patients with diseases other than SLE, and 105 healthy donors were comparatively studied to verify the initial results with this new ELISA. The cutoff was reevaluated using the results of 105 normal sera. 117 SLE

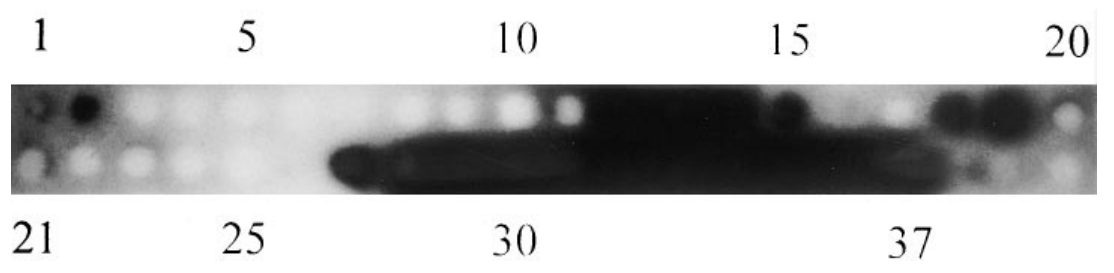

756
Figure 1. Reactivity of a representative antiSmD1 positive serum with peptides spanning the whole SmD1 protein (119 amino acids) by linear epitope mapping. Each spot represents a linear synthetic peptide of 13-aa length bound to a continuous cellulose membrane. Marked positive reactions were detected in the region of 34-52 aa (peptide 12-14) and 83-119 aa (peptide 29-37). The numbers indicate the individual peptides listed in Methods. 

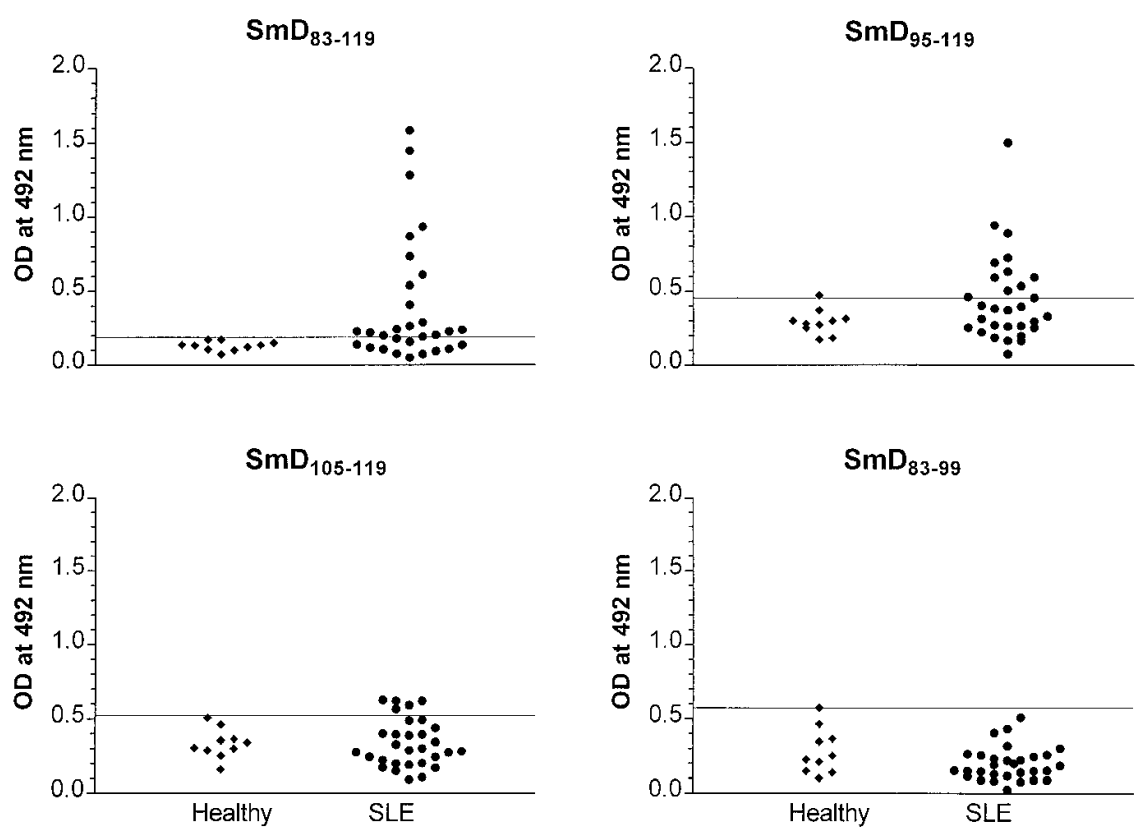

Figure 2. ELISA analysis of the SmD1 peptides aa 83-99, 95-119, 105-119 and 83-119. Anti-peptide $\operatorname{IgG}$ activity was determined in 10 normal human sera and 30 SLE sera. Antibody levels are given in OD units at $492 \mathrm{~nm}$. The cutoff line corresponds to the mean OD of 10 healthy donors +2 SD.

sera $(70.0 \%)$ were positive for anti-SmD1 $1_{83-119}$ antibodies (Fig. 3) showing a significantly elevated reactivity of up to 16,200 AU and a median level of 482 AU. Comparing these results with the response in patients with related disorders or infectious diseases with known connective tissue involvement and rheumatic complaints, there was only a low frequency and a significantly reduced reactivity in 4 of 15 SS sera (26.7\%), 5 of 23 MCTD sera (21.8\%), 4 of 20 SSc sera (20\%), 4 of 28 RA sera $(14.3 \%), 7$ of 28 PBC sera $(25 \%), 2$ of 20 hepatitis B sera ( $10 \%$, one serum was positive for antinuclear antibodies), and 5 of 30 Lyme sera $(16.7 \%)$. No healthy donor and no patient with HIV $(n=88)$ or ulcerative colitis $(n=15)$ reacted with the $S m D 1_{83-119}$ peptide. In summary, 31 of 372 control sera $(8.3 \%)$ were positive thus revealing a specificity of $91.7 \%$ for SLE in the SmD1 $1_{83-119}$ ELISA.
Anti-Sm reactivity measured by different methods. 15 SLE sera were selected according to their anti-SmD1 $1_{83-119}$ ELISA reactivities, 14 sera ranging from low to high positive and one negative serum. Comparison of the anti-SmD response revealed positive results for five sera in immunoblot with MOLT4 cell antigens, three in CIE, four in dot assay of linear epitopes, and five in a commercially available anti-Sm-ELISA with purified Sm antigens including SmD (Table II). The one negative serum in anti-SmD $1_{83-119}$ ELISA was also negative in all other assays. In contrast, only 1 of the 14 positive sera was positive in all other applied tests, and only approximately one third of initially positive sera in the SmD $1_{83-119}$ ELISA were reactive in immunoblot or CIE. Besides the high sensitivity of SmD $1_{83-119}$ ELISA due to the selection of these sera, the significantly lower detection frequency of an anti-SmD response in

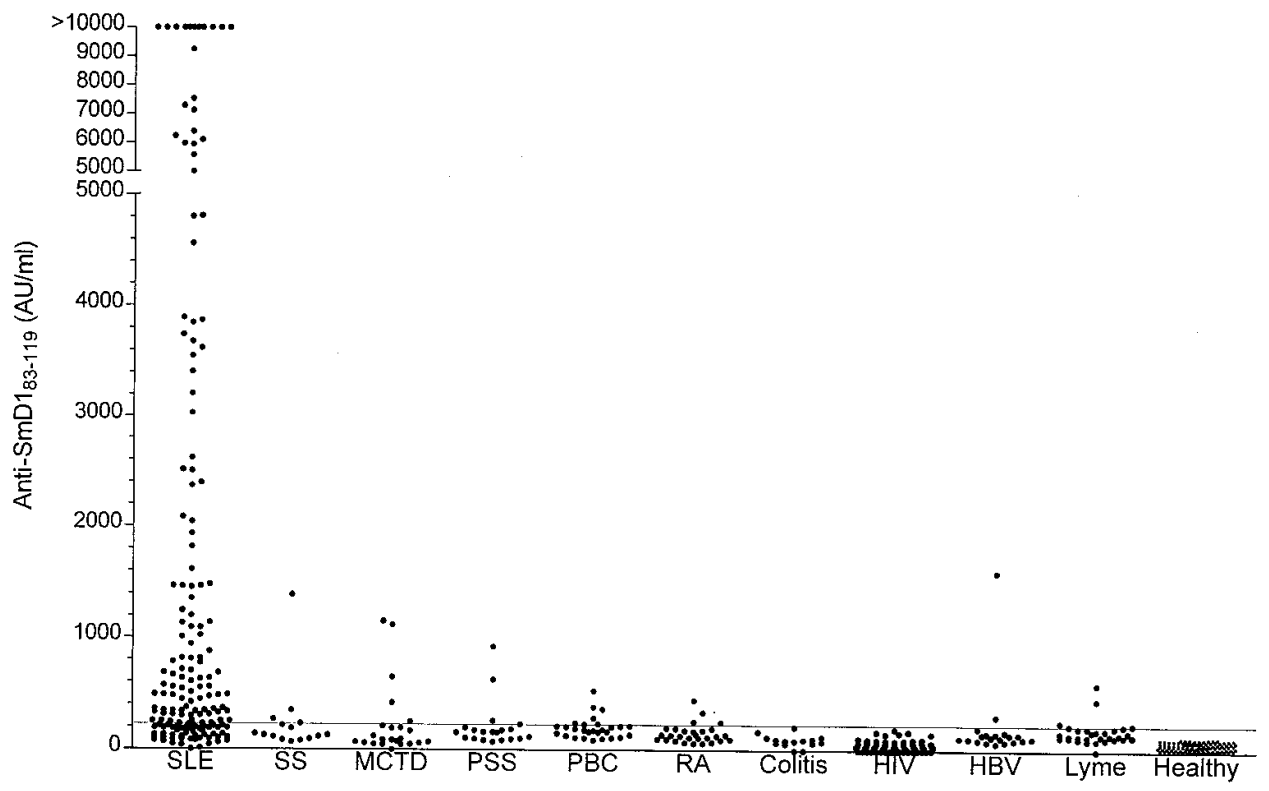

Figure 3. Anti-SmD1 $1_{83-119}$ ELISA reactivity of sera from patients with $\operatorname{SLE}(n=167)$, primary SS $(n=15)$, $\operatorname{MCTD}(n=23), \operatorname{SSc}(P S S)(n=$ $20)$, PBC $(n=28)$, RA $(n=28)$, ulcerative colitis (Colitis; $n=15$ ), HIV infection $(n=88)$, hepatitis B infection $(H B V ; n=20)$, Lyme disease $(n=30)$, and normal donors (Healthy; $n=105)$. All sera were diluted 1:100 or 1:400 if the absorbance was above the standard curve. $\mathrm{IgG}$ antibody reactivity is expressed by AUs. 


\section{Different Methods*}

\begin{tabular}{|c|c|c|c|c|c|c|c|}
\hline \multirow[b]{2}{*}{ Patients } & \multicolumn{2}{|c|}{$\begin{array}{c}\text { SmD1 }_{83-119} \\
\text { ELISA } \\
\text { reactivity }(\mathrm{AU})\end{array}$} & \multicolumn{2}{|c|}{$\begin{array}{c}\text { cSm-ELISA } \\
(\mathrm{U} / \mathrm{ml})\end{array}$} & \multirow[b]{2}{*}{ CIE } & \multirow[b]{2}{*}{ Immunoblot } & \multirow{2}{*}{$\begin{array}{l}\text { Dot } \\
\text { assay }\end{array}$} \\
\hline & Quant. & Qual. & Quant. & Qual. & & & \\
\hline 1 & 630 & pos. & 6,6 & - & pos. & pos. & - \\
\hline 2 & 5971 & pos. & 5 & - & - & - & pos..$^{\ddagger}$ \\
\hline 3 & 311 & pos. & 11,1 & pos. & - & - & - \\
\hline 4 & 10000 & pos. & 50 & pos. & pos. & pos. & - \\
\hline 5 & 192 & - & 2,8 & - & - & - & - \\
\hline 6 & 1819 & pos. & 4,3 & - & - & - & - \\
\hline 7 & 10000 & pos. & 2,9 & - & - & - & - \\
\hline 8 & 678 & pos. & 1,5 & - & - & - & - \\
\hline 9 & 1200 & pos. & 1,8 & - & - & - & pos. \\
\hline 10 & 10000 & pos. & 50 & pos. & pos. & pos. & pos. \\
\hline 11 & 2014 & pos. & 4,15 & - & - & - & - \\
\hline 12 & 643 & pos. & 0,8 & - & - & - & - \\
\hline 13 & 3888 & pos. & 34,6 & pos. & - & - & pos. \\
\hline 14 & 7523 & pos. & 50 & pos. & - & pos. & - \\
\hline 15 & 6500 & pos. & 3,9 & - & - & pos. & - \\
\hline
\end{tabular}

* Methods used included the SmD1 $1_{83-119}$ ELISA (cutoff $223 \mathrm{AU}$ ), a commercially available anti-Sm-ELISA (cSm-ELISA, cutoff 7), CIE, immunoblotting using MOLT4 cells (IB), and the dot assay for linear epitope mapping. " Sera directed against the epitope in the $\mathrm{C}$-terminus and in position aa 34-46. Dashes indicate negative test results. There was no significant correlation between cSm-ELISA and SmD1 $1_{83-119}$ ELISA using the Spearman's nonparametric correlation test.

the other test system showed only partial agreement for positive or negative results in individual patients.

Anti-SmD $1_{83-119}$ antibodies and correlation with disease activity. Fig. 4 displays a longitudinal follow-up of anti-SmD1 titers in one SLE patient representative for five others. This patient with acute onset of SLE had arthritis, butterfly rash, somnolence, serositis, and nephritis. The anti-SmD1 $1_{83-119}$ reactivity was extremely high. After a short course of high-dose methylprednisolone treatment, temporary clinical improvement was accompanied by a decrease in anti-SmD1 titers. SLE symptoms relapsed with increase of proteinuria, butterfly rash, and arthritis and anti-SmD1 $1_{83-119}$ titers passed through another peak until remission was induced by monthly intravenous cyclophosphamide bolus. The same findings were obtained in five additional individuals (data not shown), indicating that reactivity levels correlated with disease activity.

Association of anti-SmD1 $1_{83-119}$ and anti-dsDNA reactivities. 164 SLE sera studied in the SmD1 $1_{83-119}$ ELISA were also analyzed for anti-dsDNA antibodies by Crithidia luciliaeimmunofluorescence. 86 sera $(52.5 \%)$ were anti-dsDNA antibody positive including 65 of the 115 sera reactive against SmD1 $1_{83-119}$ peptide, thus indicating a higher prevalence of antiSmD1 $1_{83-119}$ than anti-dsDNA antibodies. Fig. 5 shows significantly higher reactivity against the $\mathrm{SmD} 1_{83-119}$ peptide in antidsDNA antibody positive compared with negative sera $(P<$ $0.0001)$. On the other hand, these data also demonstrate the existence of anti-SmD1 $1_{83-119}$ antibodies in the anti-dsDNAnegative subset of SLE patients. Four of the five human monoclonal anti-dsDNA antibody preparations also reacted with the $\mathrm{SmD}_{83-119}$ peptide in a range from 250 to 2,000 AU.

Competition assays. The specificity of the anti-SmD $1_{83-119}$ response for the $\mathrm{SmD}$ protein was tested by competition as- says using soluble $\mathrm{SmD}_{83-119}$ peptide, polystyrene-coupled SmD1 $1_{83-119}$ peptide, and TALON-bound recombinant SmD1 protein. The SmD1 positive serum used for these assays was shown to react predominantly with $\mathrm{SmD} 1$ but also with $\mathrm{SmD} 3$ at a lower level. Preadsorbtion with $\mathrm{SmD} 1_{83-119}$ peptide coupled on polystyrene significantly reduced the anti-SmD $1_{83-119}$ reactivity in the $\mathrm{SmD}_{83-119}$ ELISA and in the Western blot analysis. In contrast, anti-SmD3 reactivity was not significantly changed. Furthermore, there was a significant reduction of the ANA-immunofuorescence intensity using Hep2 cells by this procedure. Although soluble $\mathrm{SmD} 1_{83-119}$ peptide reduced the anti-SmD reactivity in immunoblotting (Fig. 6), high peptide concentrations $(80 \mu \mathrm{g} / \mathrm{ml})$ of soluble $\mathrm{SmD} 1_{83-119}$ peptide were needed to specifically suppress anti-SmD reactivity (data not shown). Nevertheless, in comparison with other proteins (oxypolygelatine) under same conditions, the soluble $\mathrm{SmD1}_{83-119}$ peptide specifically reduced the anti-SmD response in immunoblotting with MOLT4 cell extracts (Fig. 7). After adsorption of SLE sera to TALON-bound recombinant SmD1 protein, the anti-SmD1 $1_{83-119}$ reactivity showed a $90 \%$ reduction in the anti-SmD $1_{83-119}$ peptide ELISA and a reduction of SmD staining in immunoblotting using MOLT4 cell extracts and recombinant SmD1 protein (Fig. 8).

Anti-SmD1 $1_{83-119}$ response in rabbits. Immunization of rabbits with the SmD $1_{83-119}$ peptide successfully induced antibody responses in all animals detectable by $\mathrm{SmD} 1_{83-119}$ peptide ELISA and by immunoblot analysis with recombinant SmD1. The reactivity of the $S m D 1_{83-119}$ peptide ELISA corresponds to reactivity in immunoblotting with recombinant $\mathrm{SmD} 1$ protein, but only one rabbit serum reacted with the SmD1 protein in MOLT4 extracts by immunoblotting (Fig. 9). The reactivity of rabbit 1 was not directed against SmD1. However, there was a low reactivity against the band identical to the size of SmD3 as detected by high TEMED gelelectrophoresis and staining with polyclonal SmD3-specific rabbit sera. In immunofluorescence, sera of immunized rabbits did not show any typical ANA patterns.

\section{Discussion}

The SmD1 protein is a primary target of the autoantibodies specific for SLE (14). To study pathophysiological mechanisms of anti-Sm reactivity and to recognize important epitopes for a specific and sensitive diagnostic assay, it is necessary to know the structure and the immunodominant B cell epitopes of SmD1. After the first report of a cDNA clone encoding the SmD1 protein (16), several groups have mapped the autoantibody response against this protein by different methods, however, with inconsistent results. Using multiple fusion proteins between $\operatorname{trpE}$ and fragments of $\mathrm{SmD}$ for epitope mapping by immunoblotting, two general patterns of anti-SmD1 reactivity were described: antibodies that recognize only the full-length antigen and are presumably directed against discontinuous epitopes and antibodies that recognize the carboxy terminus of the antigen, which embodies an extended/charged structure (30). Hirakata et al. (31) tested the ability of the monoclonal anti-Sm antibody Y12 and anti-Sm sera to immunoprecipitate truncated forms of SmD1 demonstrating a major autoantigenic domain on the carboxy terminus (amino acids 85-119). Using synthetic peptides Sabbatini et al. (32) confirmed these data demonstrating seroreactivity against the C-terminal SmD sequence $95-119$ in $25 \%$ of the lupus sera. This reactivity was 

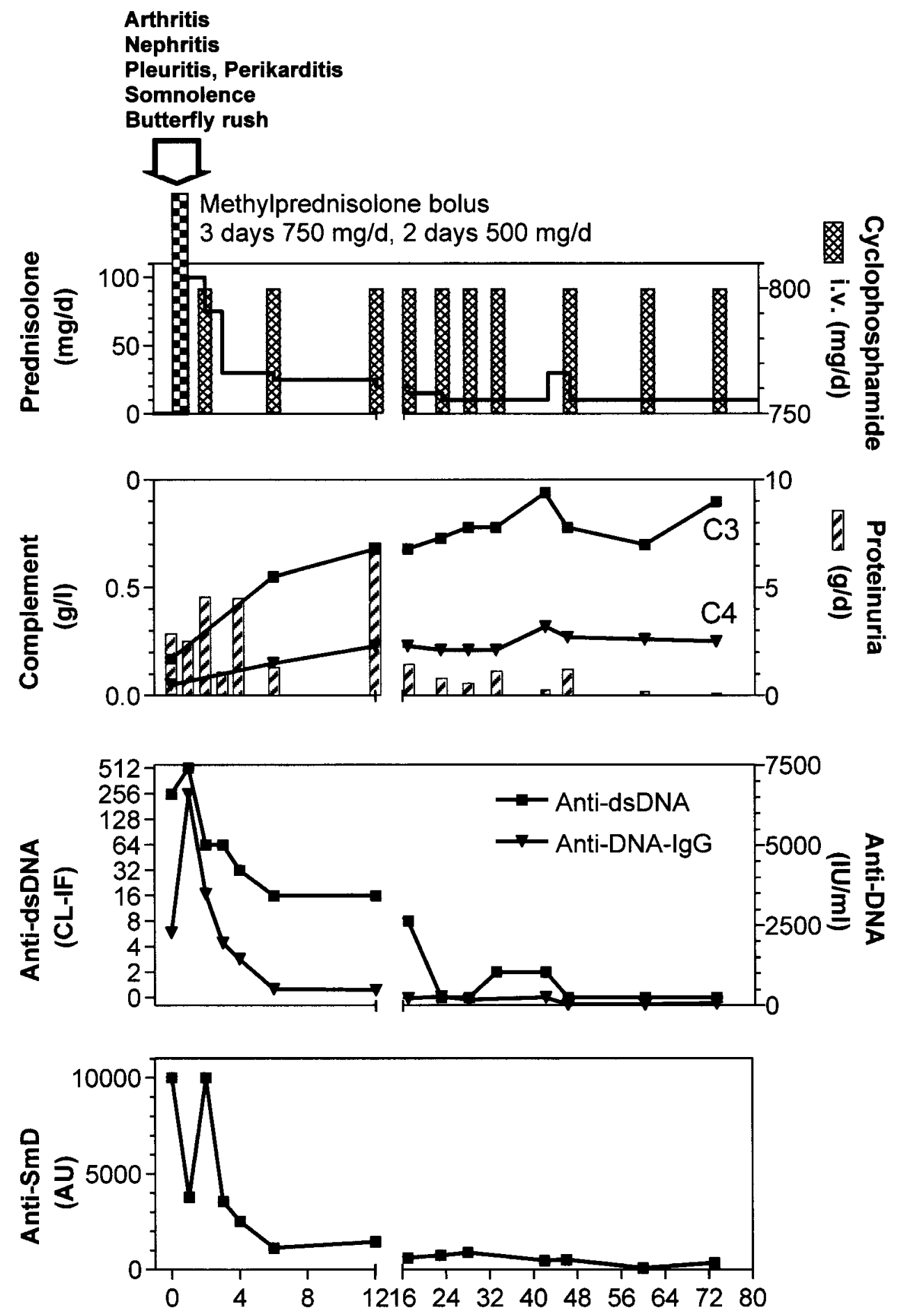

Figure 4. Course of the anti$\mathrm{SmD} 1_{83-119}$ reactivity in ELISA in correlation to disease activity in a representative SLE patient. In parallel, therapy, complement concentration, proteinuria, and reactivity of anti-dsDNA antibodies in immunofluorescence and ELISA are given.

also present by synthesizing the sequence as a multiple antigen peptide. These results were supported by others, detecting major antigenic epitopes also in the C-terminal region from 82 to 97 and 92 to 119 aa by analysis with overlapping octapeptides of the entire SmD1 protein (33). Contrasting results were found by Barakat et al. (13) who described especially a strong amino-terminal reactivity by ELISA with seven overlapping synthetic peptides.

Because of these contrasting results, we used a new method comprising simultaneous synthesis of 37 overlapping peptides covalently bound to and continuously aligned on a cellulose membrane for linear SmD1 epitope mapping. In this dot assay, two main epitopes of the anti-SmD1 response, around position 40 and at the $\mathrm{C}$-terminus, were detected. Amino-terminal reactivity as described by only one group (13) could not be confirmed. This may be due to the differences in individual peptide sequences used by these authors. Our data do not exclude that further epitopes may exist, even N-terminally, if the appropriate sequence is used. However, the fact that most groups demonstrated a C-terminal reactivity suggests this region as the major autoantigenic epitope of SmD1.

Therefore, in this study C-terminal peptides of different 


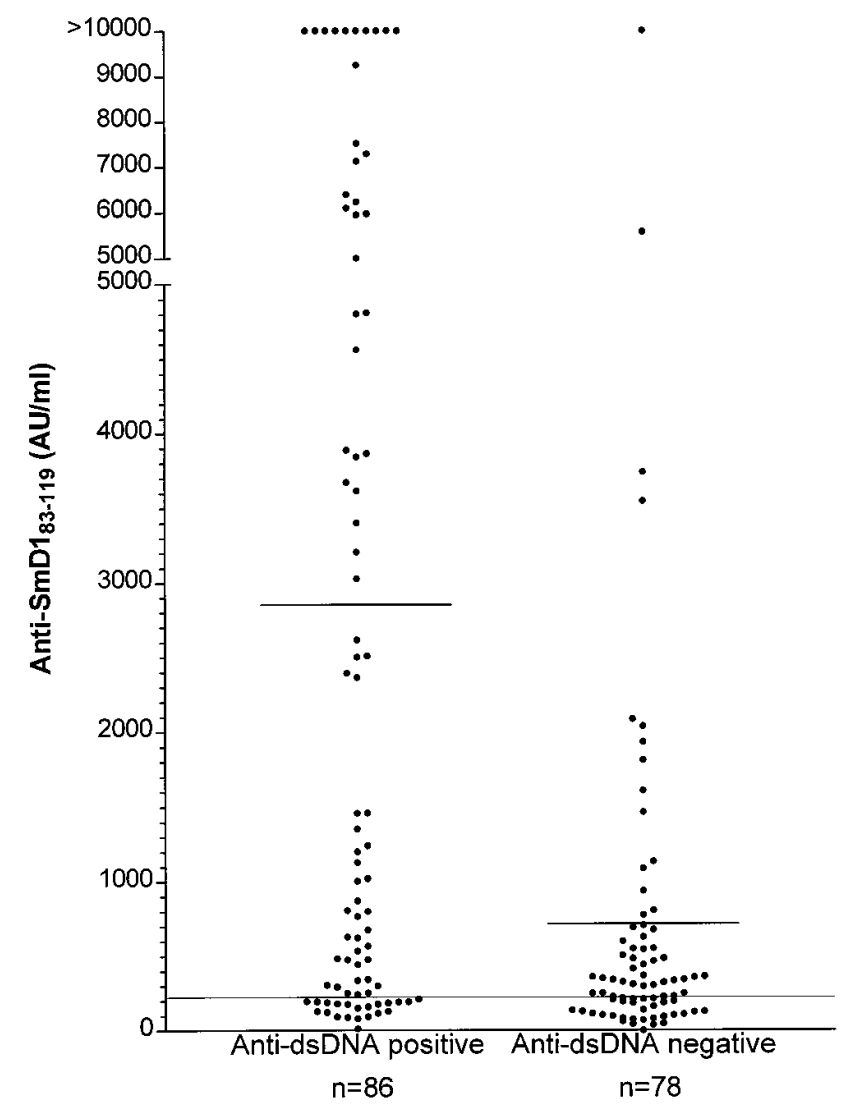

Figure 5. Demonstration of anti-SmD1 $1_{83-119}$ reactivity using ELISA in correlation to anti-dsDNA antibodies measured by Crithidia luciliae immunofluorescence. Anti-SmD1 $1_{83-119}$ reactivity was significantly increased in anti-dsDNA positive sera $(P<0.0001$, Mann-Whitney $\mathrm{U}$ test). The horizontal solid lines represent the median level of reactivity.

A) Molt4 cell extract

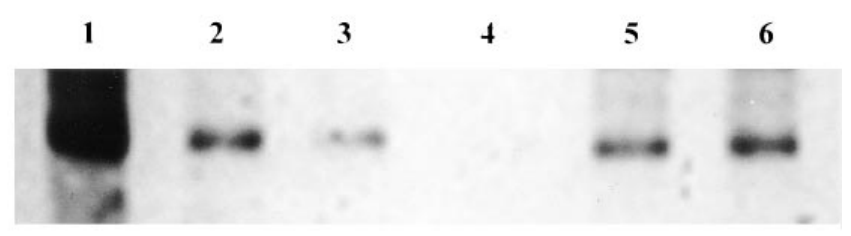

B) Recombinant SmD1

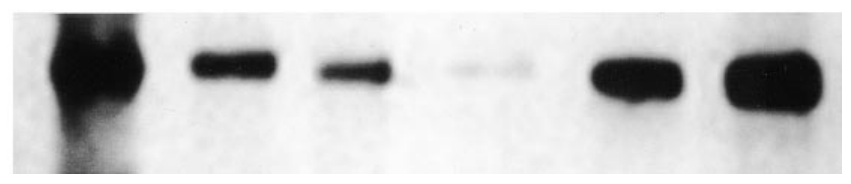

Figure 6. Inhibition of the anti-SmD1 reactivity by polystyrene bound (lanes 2-4) and soluble $\mathrm{SmD} 1_{83-119}$ peptide (lane 6) detected by immunoblotting with MOLT4 cell extracts $(A)$ and recombinant SmD1 protein $(B)$ : anti-SmD1 positive serum without inhibition (lane $1)$, sequentially incubated in three $\mathrm{SmD} 1_{83-119}$-coated microtiter wells (lane 2), five (lane 3) and eight SmD1 $1_{83-119}$-coated microtiter wells (lane 4). The anti-SmD1 reactivity decreased with repeated incubation of an anti-SmD-positive serum. As a control, serum was incubated eight times with uncoated polystyrene ELISA wells (lane 5), revealing no marked reduction of anti-SmD $1_{83-119}$ reactivity.

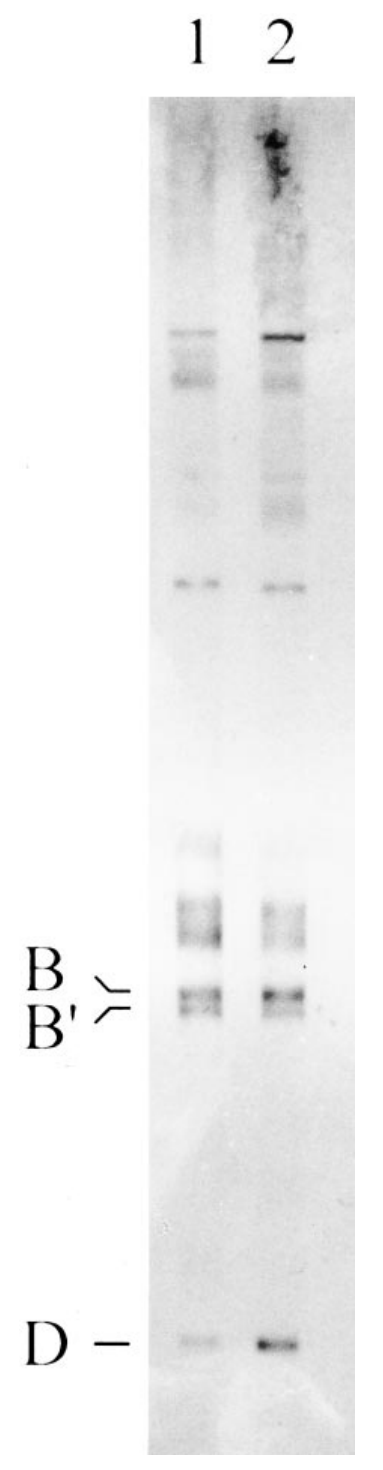

Figure 7. Inhibition of the anti-SmD1 antibody reactivity by high amounts of soluble $\mathrm{SmD} 1_{83-119}$ peptide $(80 \mu \mathrm{g} /$ $\mathrm{ml}$, lane 1) in comparison to $80 \mu \mathrm{g} / \mathrm{ml}$ oxypolygelatine (lane 2) as control. Anti-SmD1 reactivity is significantly reduced after $\mathrm{SmD} 1$ peptide incubation, whereas staining of $\mathrm{BB}^{\prime}$ is unchanged.

length were synthesized to investigate their reactivity with SLE sera by ELISA. In contrast to linear epitope mapping with 13-mer peptides, no reactivity against the peptide 83-99 was detectable by ELISA. In ELISA techniques, the peptides are adsorbed to polystyrene plates, whereas in linear epitope mapping the peptides were covalently bound to cellulose membranes. The diversity of results may be explained by different effectivity of epitope formation or different density of antigen on cellulose compared with polystyrene. With increasing length, there was a striking gain of sensitivity in detecting autoantibody reactivity in SLE. Best results were obtained using the SmD1 peptide from amino acid 83 to amino acid 119 $\left(\mathrm{SmD} 1_{83-119}\right)$. The sensitivity of anti-SmD1 $1_{83-119}$ reactivity was nearly $70 \%$ in SLE sera. This was an unexpected result, since the reported frequency of anti-Sm antibodies is lower than $10 \%$ in Caucasian SLE patients. In addition to this high sensitivity, there was no indication for loss of specificity for SLE tested in a large number of sera with different rheumatic and infectious diseases as well as healthy donors.

Reflecting the complexity of these data, the following questions arise: what is the impact of source and preparation of $\mathrm{SmD} 1$ on detection of anti-Sm reactivity, does this depend on 


\section{Molt 4 cell extract}

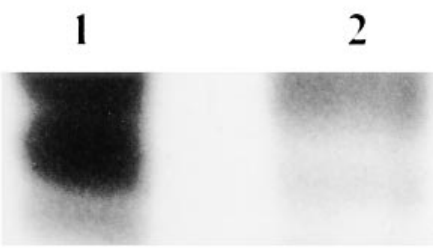

\section{Recombinant SmD1}

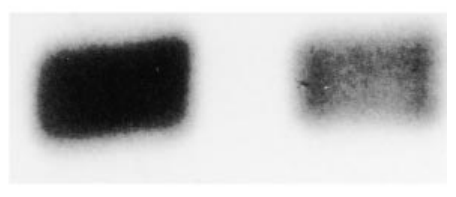

\section{Anti-SmI $)_{83-114^{-}}$ ELISA $(\mathrm{Al} / \mathrm{ml})$}

10000

1052

Figure 8. Demonstration of an inhibitory effect of recombinant SmD1 on anti-SmD1 $1_{83-119}$ reactivity. An anti-SmD1 positive serum was incubated with TALON-bound recombinant SmD1 for $1 \mathrm{~h}$ at room temperature and subsequently tested by immunoblotting using MOLT4 cell extract and recombinant $\mathrm{SmD} 1$ protein as well as antiSmD1 $1_{83-119}$ peptide ELISA (lane 2). As control, the same serum was incubated with TALON without bound recombinant SmD1 (lane 1).

folding of critical conformations, and do interactions with other nuclear molecules interfere with epitope formation?

Up to now, characterization of anti-Sm reactivity was performed on the basis of affinity-purified protein, recombinant protein from bacterial and eucaryotic sources as well as synthetic peptides. Using an affinity-purified preparation of $\mathrm{BB}^{\prime}$ and $\mathrm{D}$ proteins in a commercial anti-Sm-ELISA, there was no increase of anti-Sm detection sensitivity compared with CIE or immunoblotting. Recombinant $\mathrm{SmD} 1$ from a bacterial source, on the other hand, resulted in complete loss of anti-SmD1 binding $(34,35)$. However, recombinant SmD1 from a eucaryotic source was equally efficient as affinity-purified Sm proteins (14). Thus, this phenomenon may be explained by different posttranslational modifications in procaryotic and eucaryotic cells of a nuclear and therefore eucaryotic antigen. Nevertheless, the recombinant full-length $\mathrm{SmD} 1$ did not improve detection of anti-SmD1 (13). This seems contradictory to results presented by Rokeach et al. (16) because the conditions in their study are very different. These authors used fusion proteins between fragments of SmD1 and trpE, and the bacterial protein sequences influenced the reactivity against SmD1. Furthermore, using preselected anti-SmD positive sera as defined by immunoblotting, it can be expected that only a proportion of these sera will react to a certain fragment of the full-length SmD1. In our analysis, however, the anti-SmD1 peptide ELISA detected a larger group of Sm reactive sera in an unselected SLE population with only a small subgroup of immunoblot positive anti-SmD1 reactive sera. Therefore, the data presented in this article strongly suggest that synthetic peptides of

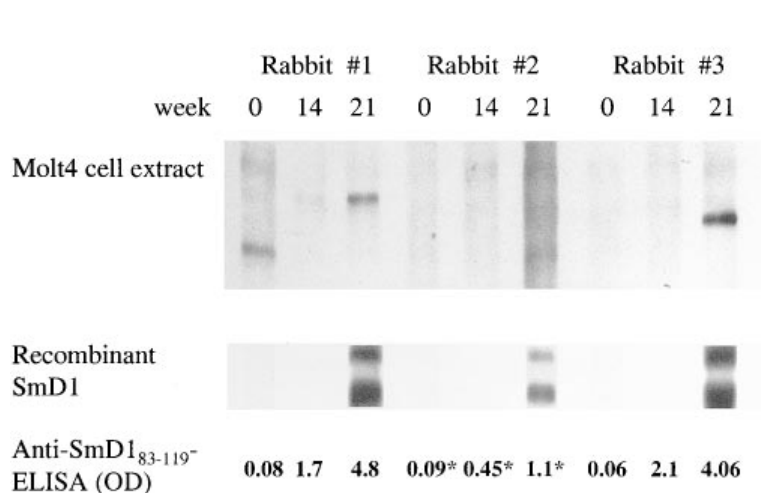

Figure 9. Polyclonal sera from three immunized rabbits were investigated by immunoblotting using MOLT4 extracts, recombinant SmD1 protein, and by anti-SmD1 ${ }_{83-119}-$ ELISA. Sera were tested before and after immunization and the reactivity was measured by OD. Because of high reactivities after immunization, sera were diluted up to $1: 20,000$, serum of rabbit \#2 only reacted in a dilution of 1:2,500 as marked by an asterisk. As control, the monoclonal antibody antiSmD1 (ICN Biomedicals GmbH, München, Germany) was tested with MOLT4-extracts. Reactivity against recombinant SmD1 was found in all three animals at week 21. Using MOLT4 extracts, only the serum from rabbit \#3 showed a weak reactivity. The reactivity in rabbit \#1 was directed against a band identical to electrophoretical size of SmD3. The monoclonal anti-SmD1 did not react with the recombinant $\mathrm{SmD} 1$ protein in the immunoblot but reacted strongly in the ELISA.

appropriate length and sequence can improve detection sensitivity significantly without loss of specificity. The superiority of synthetic peptides compared with the whole molecule for detection of anti-SmD1 reactivity is supported by other groups $(13,32)$, however, not in this magnitude.

Epitope mapping and reactivity against C-terminal peptides of different length support that conformation is crucial for anti-SmD1 reactivity. There is a striking increase of reactivity depending on length according to reports on antiSmD $1_{97-119}(13)$ and anti-SmD1 $1_{95-119}$ (32) peptides. In our study, the long peptide $S m D 1_{83-119}$ could further increase anti-SmD1 sensitivity, whereas the combination of overlapping shorter peptides, covering the same sequence length, was ineffective. On the other hand, reduced reactivity against full-length SmD1 (commercial ELISA, CIE), compared with SmD1 $1_{83-119}$ peptide as shown in Table I, suggests that the epitope formed by the peptide is cryptic. On the basis of a putative conformational epitope, circular dichroic spectroscopy was performed. According to preliminary experiments, $S m D 1_{83-119}$ peptide was found in random coil formation at least as soluble peptide (data not shown). However, proteins associated with polystyrene reportedly may fold in specific secondary structures as shown for fibronectin (36). Similar circumstances may account for our observation that the polystyrene adsorbed SmD1 $1_{83-119}$ peptide was significantly more effective in blocking anti-SmD1 specific reactivity on immunoblots compared to the soluble SmD1 $1_{83-119}$ peptide. Furthermore, antibodies may be able to induce conformational changes during the binding procedure as described for several proteins such as the acetycholine receptor (37) and viral proteins (38).

Protein folding, epitope formation, and the molecular specificity of anti-SmD1 autoantibodies may also be influenced by the interaction of native SmD1 with other molecules including 
RNA, DNA, or other proteins of the snRNP complex in vivo. As mentioned, SmE-F-G complexes or possibly other complexes of RNA-free Sm proteins may be alternative and more specific antigens in SLE (39).

Although direct interactions between DNA and Sm protein have not been demonstrated yet, overlapping reactivity has been reported by several authors (40-42). While these reports were on full-length $\mathrm{SmD}$ protein, in our experiments, there was also an overlap of reactivity against dsDNA and the anti-SmD1 $1_{83-119}$ peptide. In addition, four out of five human monoclonal anti-dsDNA antibodies were reactive with the SmD $1_{83-119}$ peptide. However, removing the anti-dsDNA reactivity by affinity chromatography onto highly purified dsDNA did not significantly reduce the anti-SmD $1_{83-119}$ reactivity (data not shown). Only preadsorption with $\mathrm{SmD}_{83-119}$ peptide abolished this reactivity in MOLT4 immunoblots as well as ELISAs. This strongly suggests that SLE sera contain autoantibodies specific for SmD1 $1_{83-119}$ peptide and independent from an interaction with dsDNA. ANA fluorescence was also diminished, although the typical coarse speckled pattern was not completely abolished. This may be explained by an additional reactivity against $\mathrm{B} / \mathrm{B}^{\prime}$ proteins of Sm or U1RNPs, which share the same staining pattern. Monoclonal antibodies would be the appropriate tools to distinguish between these different reactivities. However, the monoclonal anti-SmD1 antibodies available did neither react with $\mathrm{SmD}_{83-119}$ peptide nor with recombinant SmD1. This lack of reactivity is not unusual. Thus, it was shown that several monoclonal anti-SmD1 antibodies did not react with recombinant $\mathrm{SmD} 1$ (14).

To further characterize the anti-SmD1 $1_{83-119}$ peptide specific immune response, three rabbits were immunized. This revealed a specific response detectable by the SmD $1_{83-119}$ peptide ELISA and by immunoblot with recombinant SmD1 in all animals. In contrast, a typical anti-Sm immunofluorescence pattern on Hep2 cells could not be demonstrated. An explanation could be that the epitope(s) is not accessible for the antibodies arising after immunization with $\mathrm{SmD} 1_{83-119}$ peptide using immunofluorescence technique on Hep2 cells.

In summary, the $\mathrm{SmD} 1_{83-119}$ peptide has been identified as an important autoantigenic epitope for SLE with extraordinarily high sensitivity and disease specificity. Our data suggest that the reactivity depends on folding into a conformational epitope. This may be enhanced by interactions with polystyrene surface, nucleic acids, other nuclear proteins, or autoantibodies themselves, although a peptide specificity of the anti-SmD1 $1_{83-119}$ response was demonstrated. This epitope may reflect a natural but hidden epitope in the full-size protein. Further analysis of this complex anti-SmD1 reactivity is necessary. Immunization experiments are currently performed with regard to intermolecular epitope spreading to reveal insight into the correlation between disease activity and anti-SmD1 $1_{83-119}$ reactivity as well as into the pathogenesis of the autoimmune response in SLE.

\section{Acknowledgments}

We thank H. Welfle for circular dichroism measurements (Max Delbrück Center for Molecular Medicine, Berlin, Germany), C. Frömmel (Dept. Biochemistry, Charité) G. Keyßer (Dept. Rheumatology, Charité), E. Dogu and Dr. St. Bläß for their advice and comments.

The research was supported by grants from the Deutsche Forschungsgemeinschaft Hi 620/1-1 and from the Medical School (Charité) 96-096.

\section{References}

1. von Mühlen, C., and E.M. Tan. 1995. Autoantibodies in the diagnosis of systemic rheumatic diseases. Semin. Arthritis Rheum. 24:323-358.

2. Tan, E.M., and H.G. Kunkel. 1966. Characteristics of a soluble nuclear antigen precipitating with sera of patients with systemic lupus erythematosus. $J$. Immunol. 96:464-471.

3. Tan, E.M., A.S. Cohen, J.F. Fries, A.T. Masi, D.J. McShane, N.F. Rothfield, J.G. Schaller, N. Talal, and R.J. Winchester. 1982. The 1982 revised criteria for the classification of systemic lupus erythematosus. Arthritis Rheum. 25: 1271-1277.

4. Yasuma, M., Y. Takasaki, K. Matsumoto, A. Kodama, H. Hashimoto, and S. Hirose. 1990. Clinical significance of IgG anti-Sm antibodies in patients with systemic lupus erythematosus. J. Rheumatol. 17:469-475.

5. Takeda, Y., G.S. Wang, R.J. Wang, S.K. Anderson, I. Pettersson, S. Amaki, and G.C. Sharp. 1989. Enzyme-linked immunosorbent assay using isolated (U) small nuclear ribonucleoprotein polypeptides as antigens to investigate the clinical significance of autoantibodies to these polypeptides. Clin. Immunol. Immunopathol. 50:213-230.

6. Hiepe, F., S.T. Kiessig, K. Yamamoto, G. Prokop, F. Mielke, A. Lukowsky, B. Schlaak, T. Miyamoto, and E. Apostoloff. 1990. Detection of autoantibodies to extractable nuclear antigens in autoimmune diseases of rheumatic origin using immunoblotting-comparison with counter-electrophoresis. Z. Rheumatol. 49:304-309.

7. Abuaf, N., C. Johanet, P. Chretien, B.I. Absalon, J.C. Homberg, and J.F. Buri. 1990. Detection of autoantibodies to Sm antigen in systemic lupus erythematosus by immunodiffusion, ELISA and immunoblotting: variability of incidence related to assays and ethnic origin of patients. Eur. J. Clin. Invest. 20:354359.

8. Seraphin, B. 1995. Sm and Sm-like proteins belong to a large family: identification of proteins of the U6 as well as the U1, U2, U4 and U5 snRNPs. EMBO J. 14:2089-2098.

9. Lerner, M.R., J.A. Boyle, S.M. Mount, S.L. Wolin, and J.A. Steitz. 1980. Are snRNPs involved in splicing? Nature. 283:220-224.

10. Krämer, A. 1996. The structure and function of proteins involved in mammalian pre-mRNA splicing. Annu. Rev. Biochem. 65:367-409.

11. Lehmeier, T., K. Foulaki, and R. Lührmann. 1990. Evidence for three distinct D proteins, which react differentially with anti-Sm autoantibodies, in the cores of the major snRNPs U1, U2, U4/U6 and U5. Nucleic Acids Res. 18: 6475-6484.

12. Lerner, M.R., and J.A. Steitz. 1979. Antibodies to small nuclear RNAs complexed with proteins are produced by patients with systemic lupus erythematosus. Proc. Natl. Acad. Sci. USA. 76:5495-5499.

13. Barakat, S., J.P. Briand, J.C. Weber, and S. Muller. 1990. Recognition of synthetic peptides of Sm-D autoantigen by lupus sera. Clin. Exp. Immunol. 81: 256-262.

14. Ou, Y., D. Sun, G.C. Sharp, and S.O. Hoch. 1997. Screening of SLE sera using purified recombinant Sm-D1 protein from a baculovirus expression system. Clin. Immunol. Immunopathol. 83:310-317.

15. Habets, W.J., D.J. de Rooij, M.H. Hoet, L.B. van de Putte, and W.J. van Venrooij. 1985. Quantitation of anti-RNP and anti-Sm antibodies in MCTD and SLE patients by immunoblotting. Clin. Exp. Immunol. 59:457-466.

16. Rokeach, L.A., J.A. Haselby, and S.O. Hoch. 1988. Molecular cloning of a cDNA encoding the human Sm-D autoantigen. Proc. Natl. Acad. Sci. USA. $85: 4832-4836$

17. Arnett, F.C., S.M. Edworthy, D.A. Bloch, D.J. McShane, J.F. Fries, N.S. Cooper, L.A. Healey, S.R. Kaplan, M.H. Liang, H.S. Luthra, et al. 1988. The American Rheumatism Association 1987 revised criteria for the classification of rheumatoid arthritis. Arthritis Rheum. 31:315-324.

18. Anonymous. 1980. Preliminary criteria for the classification of systemic sclerosis (scleroderma). Subcommittee for scleroderma criteria of the American Rheumatism Association Diagnostic and Therapeutic Criteria Committee. Arthritis Rheum. 23:581-590.

19. Winkler, T.H., H. Fehr, and J.R. Kalden. 1992. Analysis of immunoglobulin variable region genes from human IgG anti-DNA hybridomas. Eur. J. Immunol. 22:1719-1728.

20. Kramer, A., E.R. Volkmer, R. Malin, U. Reineke, and M.J. Schneider. 1993. Simultaneous synthesis of peptide libraries on single resin and continuous cellulose membrane supports: examples for the identification of protein, metal and DNA binding peptide mixtures. Pept. Res. 6:314-319.

21. Atherton, E. 1989. Solid Phase Peptide Synthesis: A Practical Approach. IRL Press, Oxford.

22. Kurata, N., and E.M. Tan. 1976. Identification of antibodies to nuclear acidic antigens by counterimmunoelectrophoresis. Arthritis Rheum. 19:574-580.

23. Hiepe, F., S.T. Kiessig, S. Jahn, H.D. Volk, R. Grunow, E. Apostoloff, and R. von Baehr. 1986. A sensitive and class specific solid phase enzyme immunoassay for anti-DNA autoantibodies in supernatants of lymphocyte cultures and human hybridomas. Biomed. Biochim. Acta. 45:K29-K35.

24. Takeuchi, K., S.J. Turley, E.M. Tan, and K.M. Pollard. 1995. Analysis of the autoantibody response to fibrillarin in human disease and murine models of autoimmunity. J. Immunol. 154:961-971.

25. Hultman, P., S. Enestrom, S.J. Turley, and K.M. Pollard. 1994. Selective 
induction of anti-fibrillarin autoantibodies by silver nitrate in mice. Clin. Exp. Immunol. 96:285-291.

26. Mullis, K., F. Faloona, S. Scharf, R. Saiki, G. Horn, and H. Erlich. 1986. Specific enzymatic amplification of DNA in vitro: the polymerase chain reaction. Cold Spring Harb. Symp. Quant. Biol. 51:263-273.

27. Villarejo, M.R., and I. Zabin. 1974. $\beta$-galactosidase from termination and deletion mutant strains. J. Bacteriol. 120:466-474.

28. Hochuli, E., H. Dobeli, and A. Schacher. 1987. New metal chelate adsorbent selective for proteins and peptides containing neighboring histidine residues. J. Chromatogr. 411:177-184.

29. Johnson, H.M., M.P. Langford, B. Lakhchaura, T.S. Chan, and G.J. Stanton. 1982. Neutralization of native human gamma interferon (HuIFN gamma) by antibodies to a synthetic peptide encoded by the $5^{\prime}$ end of HuIFN gamma cDNA. J. Immunol. 129:2357-2359.

30. Rokeach, L.A., M. Jannatipour, J.A. Haselby, and S.O. Hoch. 1992. Mapping of the immunoreactive domains of a small nuclear ribonucleoproteinassociated Sm-D autoantigen. Clin. Immunol. Immunopathol. 65:315-324.

31. Hirakata, M., J. Craft, and J.A. Hardin. 1993. Autoantigenic epitopes of the B and D polypeptides of the U1 snRNP. Analysis of domains recognized by the Y12 monoclonal anti-Sm antibody and by patient sera. J. Immunol. 150: 3592-3601.

32. Sabbatini, A., M.P. Dolcher, B. Marchini, S. Bombardieri, and P. Migliorini. 1993. Mapping of epitopes on the SmD molecule: the use of multiple antigen peptides to measure autoantibodies in systemic lupus erythematosus. $J$. Rheumatol. 20:1679-1683.

33. James, J.A., M.J. Mamula, and J.B. Harley. 1994. Sequential autoantigenic determinants of the small nuclear ribonucleoprotein Sm D shared by human lupus autoantibodies and MRL lpr/lpr antibodies. Clin. Exp. Immunol. 98: $419-426$.

34. Wagatsuma, M., N. Asami, J. Miyachi, S. Uchida, H. Watanabe, and E.
Amann. 1993. Antibody recognition of the recombinant human nuclear antigens RNP 70 kD, SS-A, SS-B, Sm-B, and Sm-D by autoimmune sera. Mol. Immunol. 30:1491-1498.

35. Rokeach, L.A., J.A. Haselby, and S.O. Hoch. 1992. Overproduction of a human snRNP-associated Sm-D autoantigen in Escherichia coli and Saccharomyces cerevisiae. Gene. 118:247-253.

36. Ugarova, T.P., C. Zamarron, Y. Veklich, R.D. Bowditch, M.H. Ginsberg, J.W. Weisel, and E.F. Plow. 1995. Conformational transitions in the cell binding domain of fibronectin. Biochemistry. 34:4457-4466.

37. Valenzuela, C.F., A.J. Dowding, H.R. Arias, and D.A. Johnson. 1994. Antibody-induced conformational changes in the Torpedo nicotinic acetylcholine receptor: a fluorescence study. Biochemistry. 33:6586-6594.

38. Zhou, Y.J., J.W. Burns, Y. Morita, T. Tanaka, and M.K. Estes. 1994. Localization of rotavirus VP4 neutralization epitopes involved in antibodyinduced conformational changes of virus structure. J. Virol. 68:3955-3964.

39. Brahms, H., V.A. Raker, and R. Lührmann. 1997. A major, novel systemic lupus erythematosus autoantibody class recognizes the E, F, and G Sm snRNP proteins as an E-F-G complex but not in their denatured states. Arthritis Rheum. 40:672-682.

40. Bloom, D.D., J.L. Davignon, P.L. Cohen, R.A. Eisenberg, and S.H. Clarke. 1993. Overlap of the anti-Sm and anti-DNA responses of MRL/Mp-lpr/ lpr mice. J. Immunol. 150:1579-1590.

41. Reichlin, M., A. Martin, A.E. Taylor, K. Tsuzaka, W. Zhang, M.W. Reichlin, E. Koren, F.M. Ebling, B. Tsao, and B.H. Hahn. 1994. Lupus autoantibodies to native DNA cross-react with the A and D SnRNP polypeptides. J. Clin. Invest. 93:443-449.

42. Zhang, W., and M. Reichlin. 1995. IgM anti-A and D SnRNP proteins and IgM anti-dsDNA are closely associated in SLE sera. Clin. Immunol. Immunopathol. 74:70-76. 